\title{
Prediction on Attenuation of Silencer Equipment with Many Resonators*
}

\author{
Kunihiko ISHIHARA $^{* *}$, Toru OKAMURA ${ }^{* * *}$, Yuichi MORIGUCHI ${ }^{* * *}$ \\ and Makoto WATANABE ${ }^{* * * *}$ \\ **Department of Mechanical Engineering, The University of Tokushima, \\ 2-1, Minami-Jyosanjima-cho, Tokushima, 770-8506, Japan \\ E-mail: ishihara@me.tokushima-u.ac.jp \\ ***Kawasaki HeavyIndustries, LTD., \\ ${ }^{* * * *}$ Sasakura LTD.,
}

\begin{abstract}
This paper describes the prediction on attenuation of a silencer tower with many resonators by using FEM. The purpose of this study is to realize the reduction of infra sound which is generated in various plants. The attenuation is firstly predicted by a conventional method and secondarily calculated by FEM. In modeling of FEM, optimal mesh number is determined so that the variation of the natural acoustic frequency becomes within $10 \%$ of the detailed model by the calculating with various mesh models, because the fine mesh model consumes much time to calculate. Finally, the experiment is performed and the calculated result is compared with the experimental one. As a result, it was clarified that the attenuation of a complex silencer with many resonators could be predicted by the finite element method. And the attenuation calculated by the conventional method could also be predicted by taking into account of $70 \%$ the value of the calculated result.
\end{abstract}

Key words: Environmental engineering, Noise control, Silencer, Prediction, Many Resonators, Attenuation, FEM, Infrasound, Natural acoustic Frequency

\section{Introduction}

In a gas turbine power plant and a large fast wind tunnel, an infra sound (below 20Hz) is generated due to a vortex arising in an exhaust duct and shock wave. The infra sound can't be heard but its existence is realized by shaking the window and it can produce harmful wealth effects. So it is necessary to reduce the infra sound before designing when the plant will be built.

Ishihara had the experience to design the large inserted silencer and apply it to the power plant in order to reduce the infra sound ${ }^{(1)}$. The silencer has the diameter of $12 \mathrm{~m}$ and the cave length of $10 \mathrm{~m}$ and the large reduction effect could be confirmed.

The acoustic characteristic of the infra sound is the wave length being long and the noise reduction effect of the absorbing and intercepting materials is small and the air absorption is also small. Then the countermeasure to the infra sound is generally difficult to make. However the reactive type silencer and the active noise silencer are obliged to be used because of the infra sound being problem.

The active noise silencer has been investigated for many years from the point of view of the practice in various industrial fields. The active silencer has been applied to the exhaust duct of the diesel engine with one-dimensional acoustic field ${ }^{(2)}$ and car room with

"Received 23 Jan 2007 (No. T-04-1123)

Japanese Original : Trans. Jpn. Soc. Mech. Eng., Vol.71, No.709, C (2005),

pp. 2723-2730 (Received 18 Oct., 2004) [DOI: 10.1299/jee.2.655] 


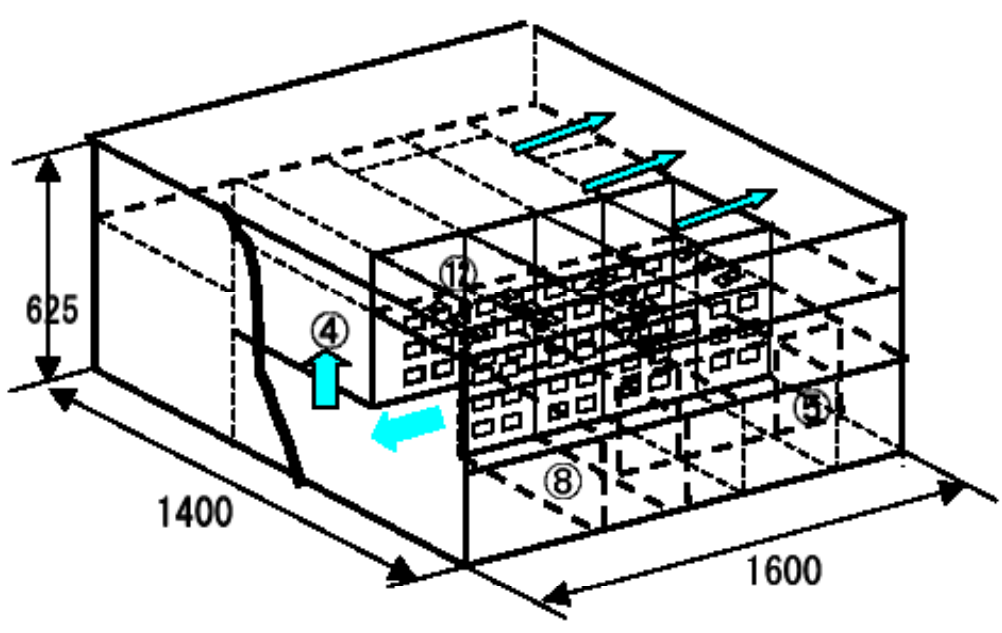

(a) Bird eye-view of silencer equipment
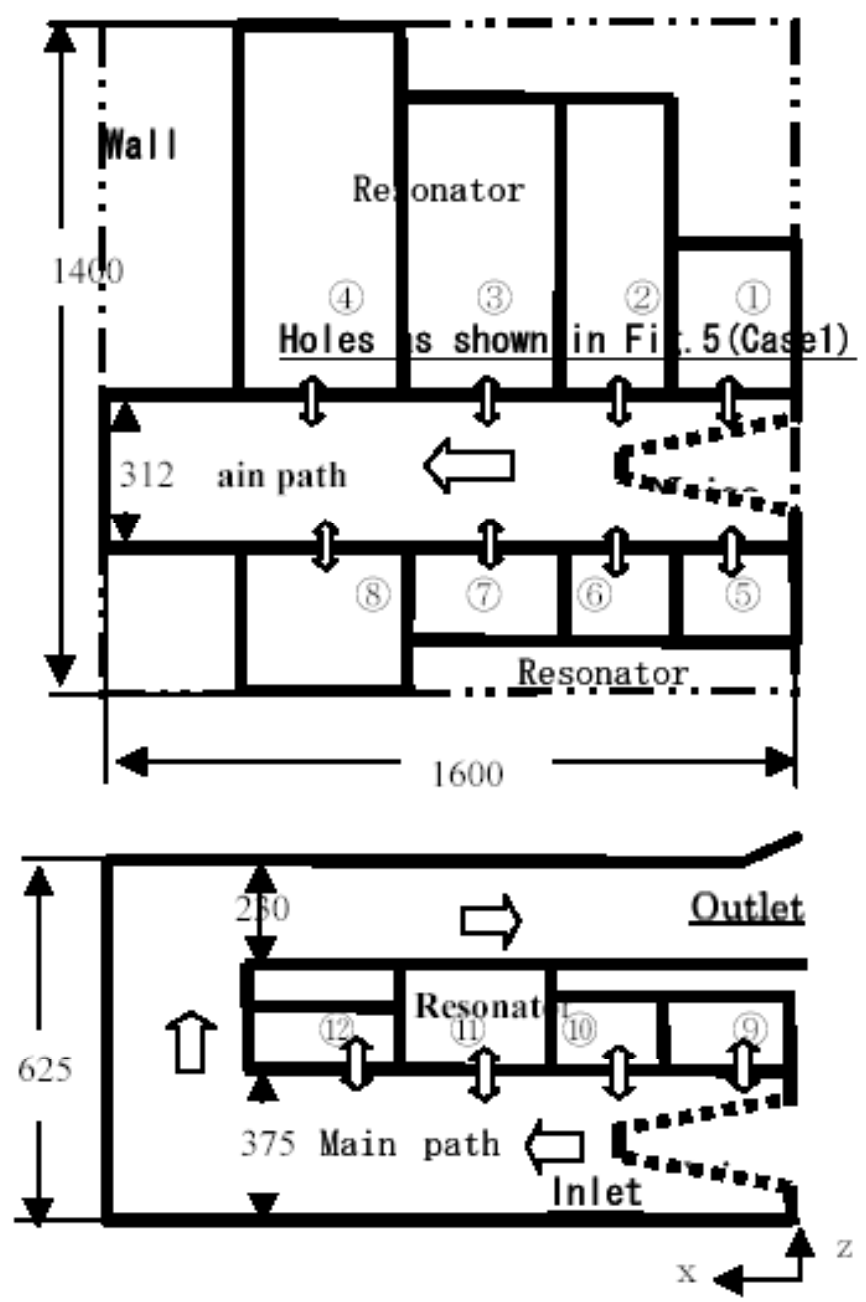

(b) Plane and front figures

Fig.1 Assumed resonant type silencer

three- dimensional acoustic field ${ }^{(3)}$. However it is the present situation that the active noise silencer has not been used as the usual countermeasure though there are many investigations $^{(4)}$

On the other hand, the reactive type silencer becomes huge due to the long wavelength as described above. So it can be considered that the silencer with many resonators of which resonant frequencies are different from each other is constructed by using the building 
already built. Such a resonator has not been applied to the actual thing yet and the reduction effect has not also been confirmed if it is ever made.

In this study, the silencer with twelve rooms of which resonant frequencies are different from each other is designed by the conventional silencer theory and its reduction effect is calculated by the finite element method after designing. The calculation result is compared with the experimental one. As a result, it is clarified that the reduction effect of the complex silencer could be predicted with the engineering accuracy by the finite element method calculation.

\section{Designing due to conventional theory and its reduction effect}

\subsection{Outline of silencer theory}

In this study, presuming the silencer to reduce the infra sound below $10 \mathrm{~Hz}$, the $1 / 32$ scale model is considered. Figure1 (a) shows the bird eye -view of the silencer equipment under consideration and figure (b) shows the plane and the front figures. The symbols (1) (12) indicate resonator rooms and they are connected with the main path at the holes(See case 1 of Fig.5) set on the boundary wall. Being given the desired resonant frequencies and reduction effects, the size of the room, the size of hole, the number of holes and the wall thickness (corresponding to neck of resonator) were determined by the following expressions ${ }^{(5),(6)}$.

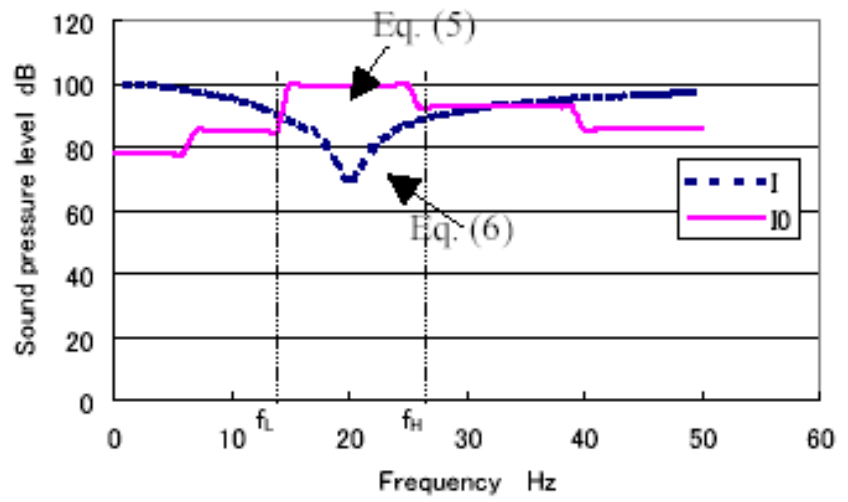

Fig.2 SPL before and after countermeasure

Table1 Calculation result based on theory

\begin{tabular}{|c|c|c|c|c|c|c|c|c|}
\hline \multirow{2}{*}{$\begin{array}{c}\text { Room } \\
\text { No. }\end{array}$} & \multicolumn{9}{|c|}{ 1/3OCT center frequency } \\
\hline & 100 & 125 & 160 & 200 & 250 & 320 & 400 & 500 \\
\hline 1 & 0 & 1 & 3 & 8 & 3 & 1 & 1 & 0 \\
\hline 2 & 2 & 5 & 10 & 3 & 1 & 1 & 0 & 0 \\
\hline 3 & 4 & 10 & 10 & 5 & 2 & 1 & 1 & 1 \\
\hline 4 & 8 & 10 & 5 & 2 & 1 & 1 & 0 & 0 \\
\hline 5 & 0 & 0 & 1 & 3 & 7 & 2 & 1 & 0 \\
\hline 6 & 0 & 0 & 1 & 5 & 2 & 1 & 0 & 0 \\
\hline 7 & 1 & 1 & 4 & 6 & 2 & 1 & 0 & 0 \\
\hline 8 & 2 & 6 & 6 & 2 & 1 & 0 & 0 & 0 \\
\hline 9 & 0 & 0 & 0 & 0 & 1 & 3 & 1 & 0 \\
\hline 10 & 0 & 0 & 0 & 0 & 1 & 4 & 1 & 0 \\
\hline 11 & 0 & 0 & 0 & 5 & 3 & 1 & 0 & 0 \\
\hline 12 & 0 & 0 & 0 & 1 & 5 & 1 & 0 & 0 \\
\hline $\mathrm{T}$ & 17 & 33 & 40 & 40 & 29 & 17 & 5 & 2 \\
\hline
\end{tabular}




$$
\begin{aligned}
& f_{r}=\frac{c}{2 \pi} \sqrt{\frac{C_{0}}{V}} \\
& C_{0}=\frac{N \times S_{0}}{l_{c}+(a \times \pi / 2)} \\
& T L(f)=10 \log \left[1+\left(\frac{\sqrt{C_{0} V} / 2 S}{f / f_{r-} f_{r} / f}\right)^{2}\right]
\end{aligned}
$$

Where the meaning of each mark are as follows.

$f_{r}$ : Resonant frequency, $V$ : Volume of cave, $c$ : Sound speed

$S:$ Cross sectional area of duct(main path), $S 0 \quad:$ Hole area

$N$ : Number of hole, $a$ : Equivalent radius of hole, $l_{c}$ : Hole length

$C_{0}$ : Conductivity, $T L(f)$ : Reduction effect

\subsection{Evaluation of reduction effect in each band width}

Equation (3) evaluates the reduction effect to the frequency continuously and shows the infinite reduction effect at the resonant frequency to tell the extremity. In actuality, the reduction effect in each frequency band is needed to know. So this equation is not suitable to apply to the reduction evaluation. Then the following evaluation expression is derived in order to obtain the reduction effect in each frequency band.

$$
T L^{i}{ }_{B}=10 \log \left(\frac{f_{i}^{H}-f_{i}^{L}}{\int_{f_{i}^{L}}^{f_{i}^{H}} 10^{-T L(f) / 10} d f}\right)
$$

Where $T L_{B}^{i}$ is the reduction effect in $i$ th band and $f_{i}^{L}, f_{i}^{H}$ are the low and the high limited frequencies respectively. In this section, this expression are conducted. On deriving, it is assumed that the intensity of sound is constant (I0) within the band as shown in Fig.2. The Sound pressure level called SPL (Lp) after through the resonator becomes like the dotted line (I) as shown in Fig.2. At this time, the SPL $L_{p 0}$ before reduction is given as follows.

$$
L_{p 0}=10 \log \frac{\int_{f^{L}}^{f^{H}} I d f}{I_{0}}
$$

And the SPL $L_{p 1}$ after reduction is given as follows.

$$
L_{p 1}=10 \log \frac{\int_{f^{L}}^{f^{H}} I \tau d f}{I_{0}}
$$

Where $\tau$ is called reduction factor temporarily. The relation between $\tau$ and $T L(d b)$ is given as follows.

$$
T L=10 \log (1 / \tau)
$$

Therefore the equivalent reduction effect can be expressed.

$$
T L_{e q}=L_{p 0}-L_{p 1}=10 \log \frac{\int_{f^{L}}^{f^{H}} d f}{\int_{f^{L}}^{f^{H}} \tau d f}
$$




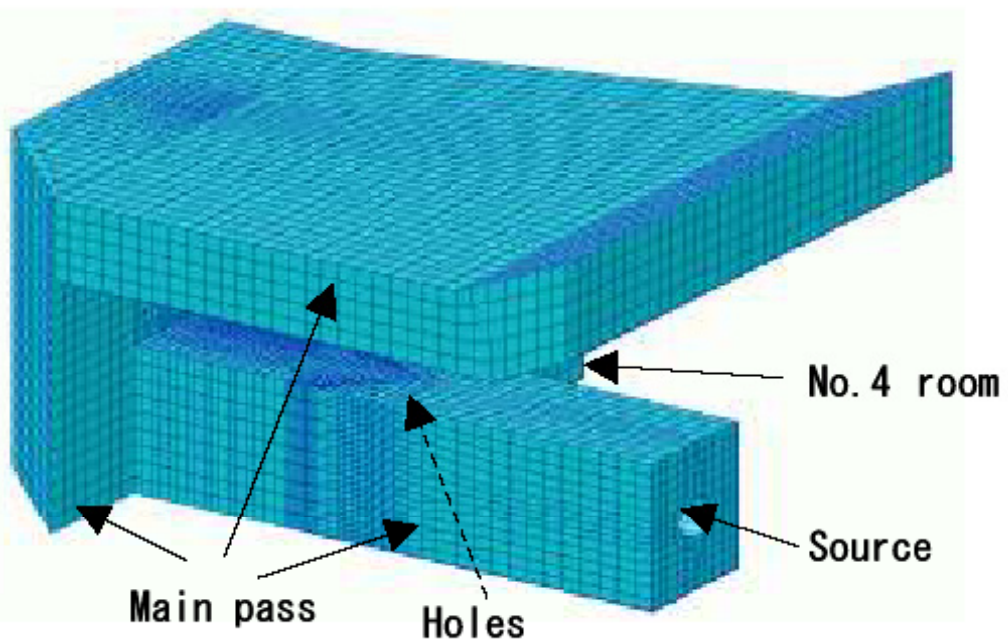

Fig.3 Analytical model (in detail)

$$
=10 \log \left(\frac{f^{H}-f^{L}}{\int_{f^{L}}^{f^{H}} 10^{-\frac{T L}{10}} d f}\right)
$$

The reduction effect obtained by this theory is shown in Table1. But the value in this table is multiplied the calculation value by 0.7

\section{Prediction of reduction effect by FEM}

As this silencer is complex and huge, the node number becomes bulky and the calculation time can not forecast when the model is meshed by following the ordinary modeling, the analysis was carried out step by step here. As the first stage, the silencer with the main path and the No.4 resonator room is modeled by the fine mesh. To put it concretely, the number of holes set on the boundary wall is made $6 \times 9$ and the mesh of each hole is made $4 \times 4$. The size of each hole is $19 \mathrm{~mm} \times 19 \mathrm{~mm}$. Next, the same model is modeled by the course mesh as the second stage and the optimal mesh is found in a manner that the variation of the resonant frequency becomes within $10 \%$ when the finite element analysis is performed. But the total hole area is the same on each meshing condition. Finally, the reasonable model is determined by taking into account the calculation time and the variation of the natural frequency, and after that the frequency response analysis is carried out. As the external force to the response analysis, the unit velocity on the nose cone shown in Fig. 1 is given.

\subsection{Calculation result of resonant frequency}

The silencer analytical model constructed by the main path and No.4 resonator room is shown in Fig.3. This is called "Fine model". The numbers of the node and element in this model are 260171 and 251748, respectively and the used program is NASTRAN. It was given as the boundary conditions that the particle velocity was zero on the wall and the sound pressure at open part was zero. The calculation time is needed $33 \mathrm{Hr}$ to obtain the only first natural frequency of this fine model as shown in Fig.4. For this reason it was clarified that this fine model was not useful to calculate. Then the hole connecting the main path and No.4 room is modeled as shown in Fig. 5 and Table 2 and we attempt to calculate the natural frequencies below $500 \mathrm{~Hz}$ on each condition. The result is shown in Table 3 . As shown in Table 3, the effect of modeling appears on the second, the third and the twelfth mode and on the contrary other modes are not subjected to the modeling effect. This is due 


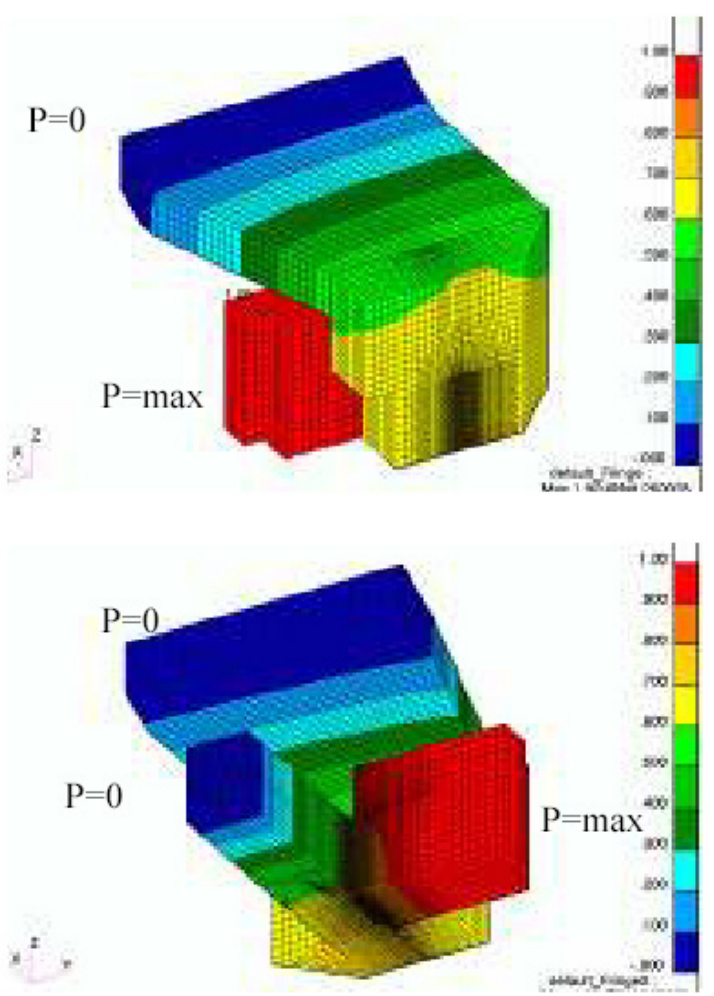

Fig. $41^{\text {st }}$ mode in detail model

Case 1

Case2

Case 3

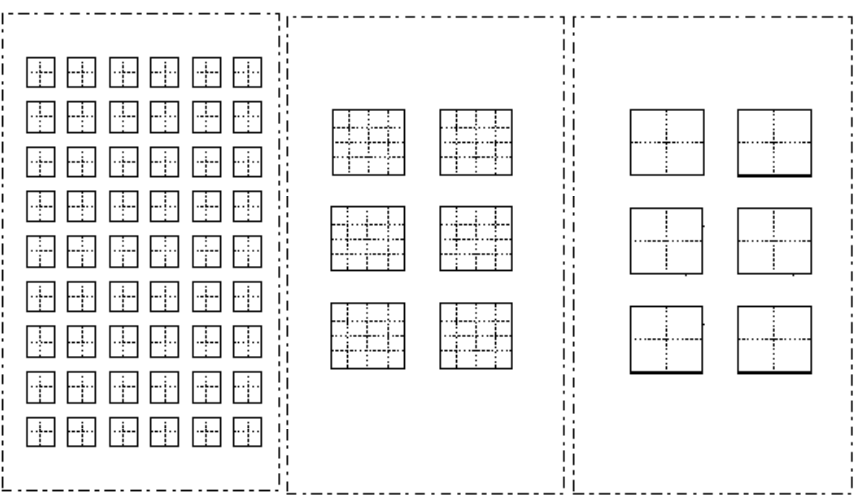

Fig. 5 Modeling of each case

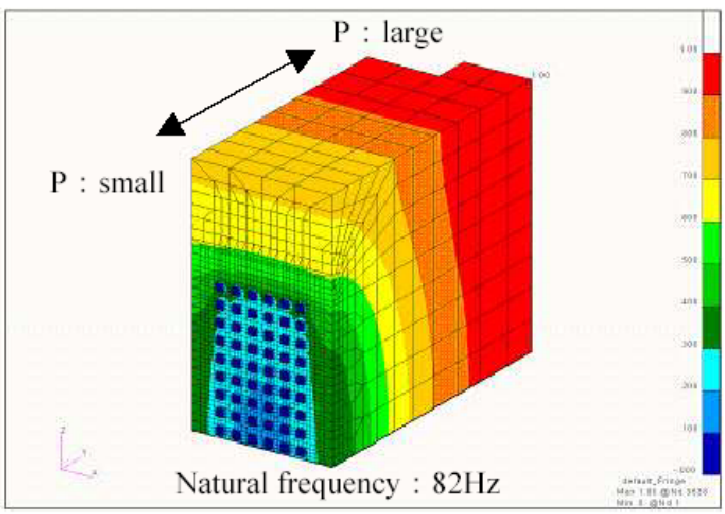

Fig.6 $1^{\text {st }}$ mode of only No.4room 
Table2 Analytical cases

\begin{tabular}{|c|c|c|c|}
\hline Case & Number of hole & Mesh number & Remarks \\
\hline 1 & $6 \times 9$ & $2 \times 2$ & \\
\hline 2 & $2 \times 3$ & $4 \times 4$ & \\
\hline 3 & $2 \times 3$ & $2 \times 2$ & \\
\hline 4 & $2 \times 3$ & $2 \times 2$ & $15 \%$ reduced of $\mathrm{t}$ \\
\hline
\end{tabular}

to the fact that the mode subjected to the modeling effect strongly is the mode cross coupled with the main path and the NO.4 resonator room and other modes are the same as the modes of only main path. Among modes subjected to the modeling effect, the second mode $(72 \mathrm{~Hz})$ is especially subjected to it strongly. This is due to the fact that the value $(72 \mathrm{~Hz})$ is close to the resonant frequency $(82 \mathrm{~Hz})$ of the resonator of No.4 room only. Comparing the relation between the number of nodes of hole (hole number $\times$ mesh number per a hole) and the natural frequency in each case, the difference of 3\% appears in mesh change (4 times) and $8 \%$ in hole number change ( 9 times) respectively. The former is the comparison of case 2 and case 3 and the latter is the comparison of case 1 and case 3 . Then the case 3 was selected as the optimal mesh model. Moreover in case 4 which the neck length is shorter than that of the case 3 , we attempt to calculate expecting the different results. But both results were the same contrary to expectations. This is due to the fact that the change of the neck length influences the natural frequency very little because of the equivalent neck length not being

Table3 Calculation results of $\mathrm{fn}$ in various cases

\begin{tabular}{|c|c|c|c|c|}
\hline Order of Mode & $\begin{array}{c}\text { Casel } \\
\text { Frequency }(\mathrm{Hz})\end{array}$ & $\begin{array}{c}\text { Case } 2 \\
\text { Frequency }(\mathrm{Hz})\end{array}$ & $\begin{array}{c}\text { Case } 3 \\
\text { Frequency }(\mathrm{Hz})\end{array}$ & $\begin{array}{c}\text { Case4 } \\
\text { Frequency }(\mathrm{Hz})\end{array}$ \\
\hline 1 & 36.99 & 36.42 & 36.03 & 36.12 \\
\hline 2 & 72.19 & 68.69 & 66.67 & 67.08 \\
\hline 3 & 122.51 & 119.73 & 118.33 & 118.61 \\
\hline 4 & 143.20 & 142.53 & 142.17 & 142.21 \\
\hline 5 & 167.81 & 167.81 & 167.81 & 167.81 \\
\hline 6 & 197.10 & 196.74 & 196.56 & 196.60 \\
\hline 7 & 217.49 & 217.47 & 217.48 & 217.48 \\
\hline 8 & 246.45 & 245.97 & 245.87 & 245.95 \\
\hline 9 & 261.64 & 260.08 & 259.16 & 259.35 \\
\hline 10 & 271.91 & 271.78 & 271.72 & 271.73 \\
\hline 11 & 273.61 & 273.03 & 272.94 & 272.97 \\
\hline 12 & 293.23 & 288.23 & 285.56 & 286.05 \\
\hline 13 & 298.86 & 298.61 & 298.56 & 298.56 \\
\hline 14 & 314.83 & 314.78 & 314.78 & 314.78 \\
\hline 15 & 342.71 & 342.69 & 342.68 & 342.68 \\
\hline 16 & 365.67 & 365.65 & 365.66 & 365.67 \\
\hline 17 & 369.82 & 368.74 & 368.47 & 368.61 \\
\hline 18 & 390.31 & 388.16 & 386.69 & 386.77 \\
\hline 19 & 394.66 & 392.53 & 392.12 & 392.18 \\
\hline 20 & 407.08 & 406.75 & 406.65 & 406.68 \\
\hline 21 & 428.69 & 428.59 & 428.54 & 428.55 \\
\hline 22 & 435.39 & 435.67 & 435.36 & 435.36 \\
\hline 23 & 443.39 & 443.26 & 443.20 & 443.20 \\
\hline 24 & 453.10 & 451.45 & 450.47 & 450.69 \\
\hline 25 & 458.47 & 458.39 & 458.36 & 458.36 \\
\hline 26 & 467.24 & 467.11 & 467.04 & 467.05 \\
\hline 27 & 473.35 & 473.12 & 473.05 & 473.07 \\
\hline 28 & 488.92 & 488.93 & 488.93 & 488.94 \\
\hline 29 & 498.38 & 499.15 & 499.88 & 499.94 \\
\hline
\end{tabular}


changed due to the wide area of hole. Namely the equivalent neck length is expressed as $L_{e}=t+\alpha(\alpha=(\pi / 2) \cdot \sqrt{S / \pi})$ and the second term is dominant. In models of case1 and case2 (or case3), the total areas are the same but different from the area per one hole. If Eqs. (1) and (2) are applied to both model, the difference of natural frequencies arises as follows

$\sqrt{l_{e q 1} / l_{\text {eq } 2}}=\sqrt{26.21 / 59.87}=0.66$

However such a large difference could not be seen in Table 3. Where eqi 1 is the equivalent neck length. Then it is considered that Eq. (2) can not be applied to the large hole like case2.

\subsection{Analytical result of reduction effect (Insertion loss)}

Next, the reduction effect of the case3 is shown in Fig.7. Where the attenuation is defined as follows.

$\mathrm{ATT}=(\mathrm{SPL}$ at exit of duct without resonators)-(SPL at exit of duct with resonators)

As the SPL at the exit duct has the distribution a little, the power average of six points as shown in Fig.8 is used as the representative value.

The cross sections $\mathrm{C}$ and $\mathrm{D}$ are located as shown in Fig.8. The attenuations at both cross sections $(\mathrm{C}$ and $\mathrm{D})$ are almost the same as seen in Fig.7. Attention to the result below $150 \mathrm{~Hz}$, the reduction effect appears at 46, 78, 108 and 140Hz. On the contrary, the minus effect (amplified effect) appears at 36, 72, 122 and 143Hz. As seen in Table4, the frequencies 46,

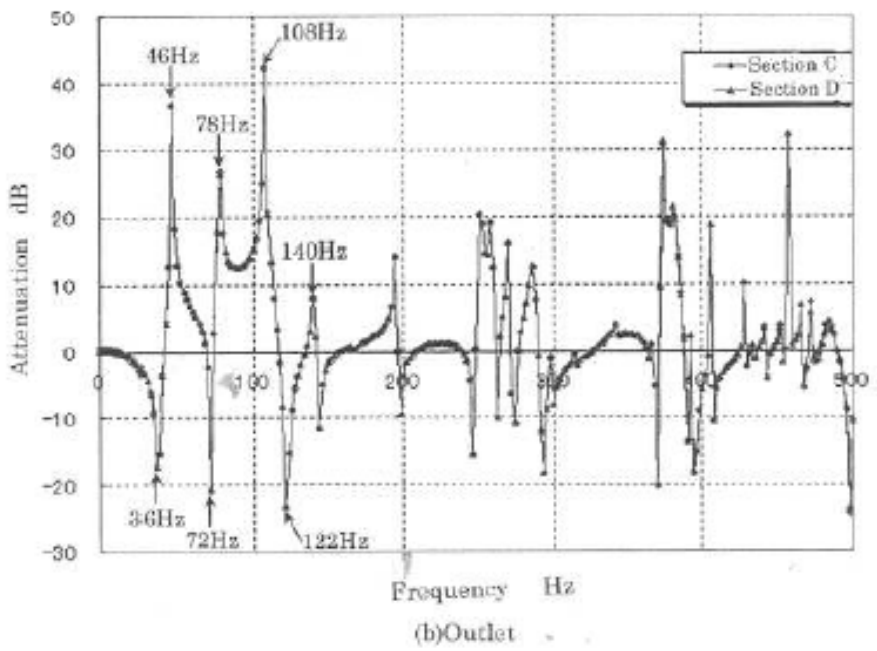

Fig.7 Reduction effect of No.4room

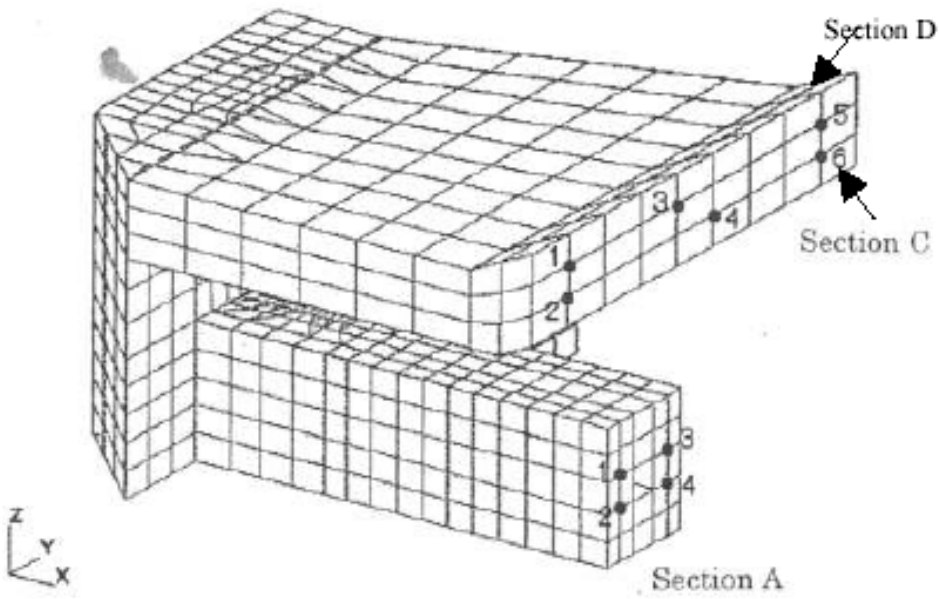

Fig.8 Evaluation points 
Table4 Natural frequency of only main duct

\begin{tabular}{|c|c|}
\hline Order of Mode & Frequency $(\mathrm{Hz})$ \\
\hline 1 & 46.86 \\
\hline 2 & 109.18 \\
\hline 3 & 143.06 \\
\hline 4 & 169.50 \\
\hline
\end{tabular}

Table 5 Resonance frequencies of final model

\begin{tabular}{|c|c|c|c|c|c|}
\hline $\begin{array}{c}\text { Order of } \\
\text { Mode }\end{array}$ & Frequency & $\begin{array}{c}\text { Order of } \\
\text { Mode }\end{array}$ & Frequency & $\begin{array}{c}\text { Order of } \\
\text { Mode }\end{array}$ & Frequency \\
\hline 1 & 30.94 & 19 & 260.00 & 37 & 385.53 \\
\hline 2 & 59.60 & 20 & 265.07 & 38 & 391.08 \\
\hline 3 & 69.30 & 21 & 272.06 & 39 & 404.16 \\
\hline 4 & 79.68 & 22 & 276.79 & 40 & 408.87 \\
\hline 5 & 89.19 & 23 & 280.85 & 41 & 427.65 \\
\hline 6 & 104.64 & 24 & 286.62 & 42 & 433.52 \\
\hline 7 & 117.39 & 25 & 298.20 & 43 & 439.28 \\
\hline 8 & 128.48 & 26 & 299.54 & 44 & 449.89 \\
\hline 9 & 132.52 & 27 & 306.68 & 45 & 454.54 \\
\hline 10 & 134.35 & 28 & 314.24 & 46 & 458.94 \\
\hline 11 & 163.39 & 29 & 326.05 & 47 & 464.21 \\
\hline 12 & 167.85 & 30 & 336.56 & 48 & 478.70 \\
\hline 13 & 170.48 & 31 & 343.01 & 49 & 480.89 \\
\hline 14 & 199.37 & 32 & 344.45 & 50 & 485.74 \\
\hline 15 & 201.40 & 33 & 346.89 & 51 & 487.52 \\
\hline 16 & 211.41 & 34 & 349.47 & 52 & 489.25 \\
\hline 17 & 217.53 & 35 & 365.96 & 53 & 492.54 \\
\hline 18 & 247.82 & 36 & 369.95 & 54 & 495.57 \\
\hline
\end{tabular}

108 and $140 \mathrm{~Hz}$ among the frequencies giving effect are the first, the second and the third natural frequencies of only the main path and the $78 \mathrm{~Hz}$ is close to the natural frequency of only the resonator shown in Fig.6. And four frequencies with minus effect are the first, the second, the third and the fourth modes of the total system (Main path + No.4 room). In case of the boundary condition being given like the sound pressure is zero at the inlet and the exit, the attenuation seems to arise at the natural frequencies of only the main path because only the main path has the natural frequency.

As described above, the frequency giving the effect becomes $78 \mathrm{~Hz}$ and this value is $66 \%$ of the value $(118.4 \mathrm{~Hz})$ calculated by Eqs. (1) and (2). This reason is considered to be as follows. These equations are available to the resonator regarded as the one degree of freedom system. Where the neck acts as the mass and the volume acts as the stiffness. Then the sound pressure is assumed to be the same everywhere in the volume. However the sound pressure distribution can be seen in Fig.6. This model can not satisfy the assumption of one degree of freedom due to the room length being over a quarter wave length. So the equivalent stiffness becomes small. In order to confirm this finding, another model constructed by the main path and the No.2 resonator room was analyzed by the finite element method. The result is $112 \mathrm{~Hz}$ and this value was $72 \%$ of the value $(154 \mathrm{~Hz})$ calculated by Eq. (1). In the resonator with the length of a quarter wavelength of resonant frequency, it should be noticeable that the real resonant frequency becomes about $70 \%$ of the value calculated by Eq. (1).

\subsection{Analytical result of attenuation}

The resonant frequency of the final model (Main path + twelve resonators) was calculated and its result is shown in Table5. There were fifty four resonant frequencies 


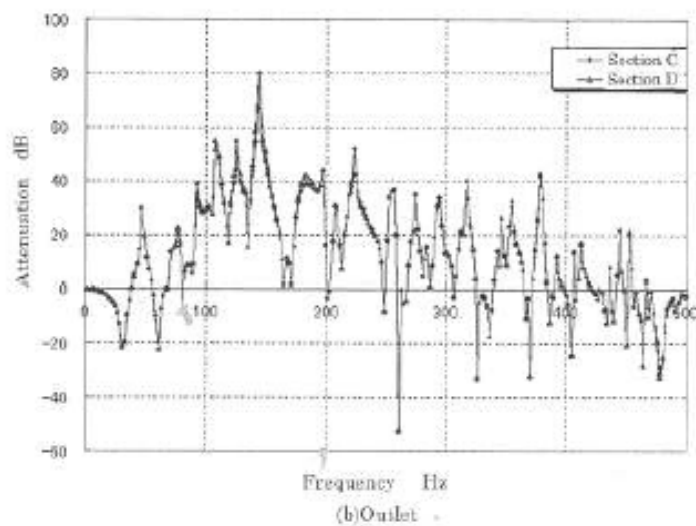

Fig.9 Reduction effect of total system(every $2 \mathrm{~Hz}$ )

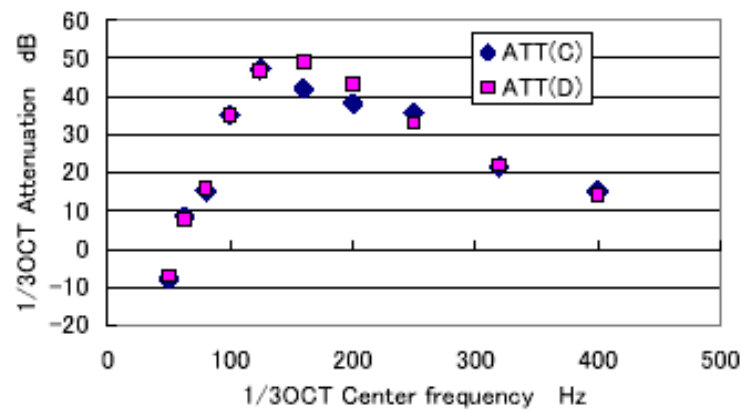

Fig.10 Reduction effect of total system(1/3OCT)

below $500 \mathrm{~Hz}$. The attenuation every $2 \mathrm{~Hz}$ and its $1 / 3$ octave band attenuation are shown in Fig.9 and Fig.10 respectively. The sound pressure level is power averaged at six points as shown in Fig.8. After that, the 1/3 octave value is recalculated and the difference of with and without resonator is obtained. This is the $1 / 3$ octave expression of attenuation. The translation from the narrow band to the $1 / 3$ octave band was performed as follows.

First, the center frequency is determined like $f_{i+1} / f_{i}=2^{1 / 3} \quad\left(\mathrm{f}_{1}=50 \mathrm{~Hz}\right)$ and the low and high limited frequencies of each band are obtained like $f_{i}^{L}=2^{-1 / 6} f_{i}, f_{i}^{H}=2^{1 / 6} f_{i}$. After obtaining the calculation number i $\mathrm{N}$ existing between $f_{i}{ }^{L}$ and $f_{i}{ }^{H}$ the $1 / 3$ octave sound pressure was calculated as follows.

$$
L_{p i}=10 \log \left(\sum_{j=1}^{N_{i}} 10^{\frac{L_{p j}}{10}}\right)
$$

\section{Comparison with experiment and consideration}

Figure 11 shows the outline of the experimental apparatus. The silencer models A and B were made of wood. The model A has no holes on the boundary wall connecting the main path and the resonator rooms. On the contrary, the model B has them. These models are set in the reverberant room as shown in Fig. 11 . The pink noise $(50 \sim 8000 \mathrm{~Hz})$ was generated by the speaker and was taken into the inlet of the silencer. The sound pressure levels in the reverberant room are measured by the two microphones (Mic.1 and Mic.2) set in the room and the frequency analysis and the averaged treatment is performed to obtain the $1 / 3$ octave band sound pressure level. After that, the difference of the results of model (A) and (B) is obtained as the attenuation. At this time, the sound pressure at the center of the duct is measured by the Mic. 3 and the experiment was carried out by confirming the input power 


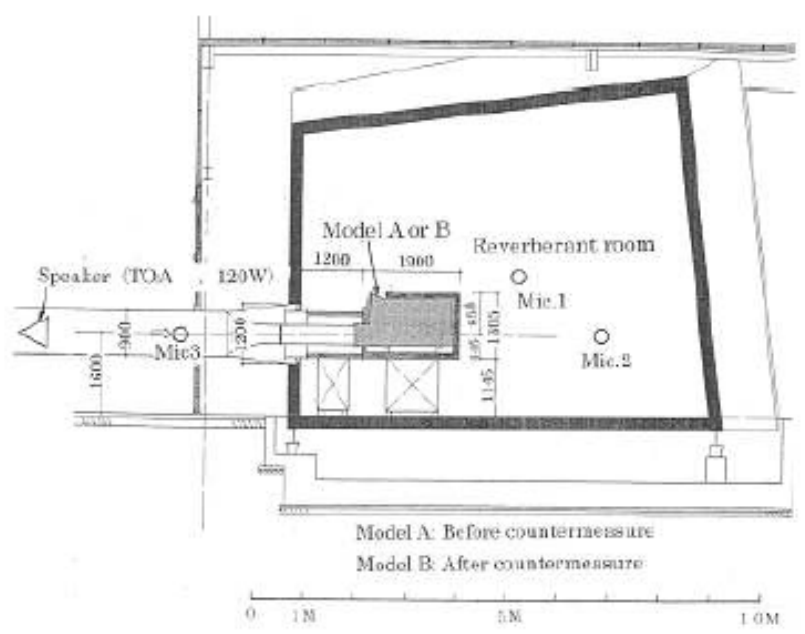

Fig11 Outline of test apparatus

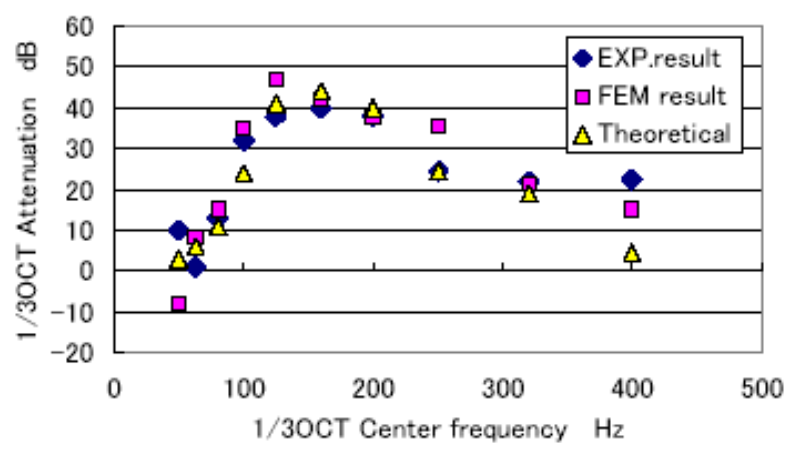

Fig.12 Comparison between calculation result and experimental ones

being the same in both models (A and B). Figure 12 shows the comparison between the analytical results by the finite element method and the experimental ones. Moreover the calculation result by the conventional theory described in Chpter2 is also plotted in Fig. 12. But this value is $70 \%$ of the calculation value. The predicted result by the finite element method was in good agreement with the experimental result and it was clarified that even the attenuation of such a complex silencer could be predicted with engineering accuracy by the finite element analysis. And it was also clarified that the conventional silencer theory was useful to the parametric study in the design stage.

Moreover, many resonators are connected with the main path at the same position longitudinally in this silencer. In this case the following problem occurs that when the attenuation of the total system is evaluated, is it reasonable to add the each attenuation simply?. The answer is as follows.

For example, the transfer matrix of the system connected with two resonators at the same position is given as follows.

$\mathrm{T}=\mathrm{T} 1 \cdot \mathrm{T} 2$

Where $\mathrm{T} 1$ and $\mathrm{T} 2$ are the transfer matrices of each resonator.

Because if two resonators are connected to the main path with length $l$, then the total transfer matrix can be given as follows.

$$
T_{1} \cdot\left[\begin{array}{cc}
\cos k l & j Z \sin k l \\
j \frac{1}{Z} \sin k l & \cos k l
\end{array}\right] \cdot T_{2}
$$


When the length $l$ becomes 0 , the second transfer matrix becomes the unit matrix.

Next, considering that many resonators are connected to the main path longitudinally at the same position, the transfer matrices of the total system can be obtained as follows.

$$
T 1 \cdot T 2 \cdots \cdot \operatorname{Tn}=\left[\begin{array}{ll}
1 & 0 \\
\sum_{i=1}^{n} \frac{1}{Z_{b i}} & 1
\end{array}\right]
$$

Where $T 1=\left[\begin{array}{cc}1 & 0 \\ \frac{1}{Z_{b 1}} & 1\end{array}\right], \cdots, T n=\left[\begin{array}{cc}1 & 0 \\ \frac{1}{Z b n} & 1\end{array}\right]$ are the driving impedance of each resonator.

This expression shows the series connection of the acoustic characteristic of the independent resonator. Therefore it can be said that the transfer matrix of the total system can be obtained by adding the each attenuation including the phase even in the case that many resonators are connected with the main path at the same position longitudinally.

\section{Conclusions}

In this study, the silencer with twelve rooms of which resonant frequencies are different each other is designed by the conventional silencer theory and its reduction effect is calculated by the finite element method after designing. The calculation result is compared with the experimental one. As a result, the following findings could be obtained.

(1) It was clarified that the attenuation of the complex silencer could be predicted with the engineering accuracy by the finite element analysis and the conventional method is useful in cases of the parametric study on the design stage and not being able to use FEM. But it is better to use $70 \%$ of the calculated one as the predicted value in that case. However it is not universal and the value obtained in comparison between the experimental result and the calculated one

(2) The meshing which is taken into account the change of the natural frequency in the finite element method is one of the reasonable methods. The attenuation can be predicted with engineering accuracy even mesh making the natural frequency within $10 \%$ variable of the accurate one.

(3) In meshing of the hole, the reasonable natural frequency can be obtained by taking one point at the center of the hole.

(4) When the length of the resonator room becomes a quarter wavelength of the resonant frequency, the real attenuation frequency becomes $70 \%$ of the theoretical value.

(5) Equation (4) or equation (8) is useful to obtain the attenuation in each frequency band.

\section{References}

(1) K. Ishihara and H. Koike, Application of Silencer for Low Frequency on Plant, Proceedings of the $6^{\text {th }}$ Simposium on Environmental Engineering (1996), pp.42-45

(2) K. Fukuyama, Diesel Engine, The Journal of INCE/J,Vol.20, No.6 (1996),pp.32-34

(3) K. Muraoka, M. Doi and T. Hamabe, Active Noise Control of Car Room, The Journal of INCE/J,Vol.20, No.6 (1996),pp.18-20

(4) M. Nishimura, Subjects and view on ANC Realization, The Journal of INCE/J,Vol.20, No.6 (1996),pp.3-6

(5) H. Shiraki et.al, Noise Protection Design and Simulation, OUYOU GIZYUTU SYUPPAN, (1987), p.20

(6) M. Fukuda and J. Okuda, Noise Countermeasure and Silencer Design, KYORITSU SYUPPAN, (1973), p.198 